\title{
A review for the automatic methods of plant's leaf image segmentation
}

\section{Shivalika Sharma and Abhishek Gupta*}

School of Computer Science and Engineering,

Shri Mata Vaishno Devi University,

Kakryal, Katra-182332, Jammu and Kashmir, India

Email:17mms010@smvdu.ac.in

Email: abhishekgupta10@yahoo.co.in

*Corresponding author

\begin{abstract}
Various plant species had disappeared and many are on the verge of disappearing. Our economy highly depends on agricultural productivity. Plants play an important role in the agriculture field. Plant's leaf segmentation has become an important task in the agricultural fields. In the leaf segmentation process, a few generalised steps are followed such as leaf image acquisition, leaf image pre-processing, feature extraction and leaf image segmentation. It is important to identify and differentiate various features from a noisy background. Feature extraction is the intermediary step to identify the features in leaves such as leaf area, leaf colour, leaf texture, etc. There are various techniques used for both feature extraction as well as segmentation of leaves like edge detection technique, K-means clustering techniques, artificial neural network-based techniques, etc. This paper presents a review of some methods used for the segmentation of leaf based on the available literature.
\end{abstract}

Keywords: leaf segmentation; feature extraction; classification; genotype trait; plant's leaf.

Reference to this paper should be made as follows: Sharma, S. and Gupta, A. (2020) 'A review for the automatic methods of plant's leaf image segmentation', Int. J. Intelligence and Sustainable Computing, Vol. 1, No. 1, pp.101-114.

Biographical notes: Shivalika Sharma has completed her MTech from Shri Mata Vaishno Devi University, Katra, Jammu and Kashmir, India. Also, she has completed her Btech from Model Institute of Engineering and Technology, Kot Bhalwal, Jammu, India. Her area of interest is digital image processing.

Abhishek Gupta has completed his BE in Computer Engineering from Rajasthan University, ME in Computer Science and Engineering from PEC University of Technology, Chandigarh and $\mathrm{PhD}$ in Engineering from Academy of Scientific and Innovative Research (AcSIR) at CSIR-Central Scientific Instruments Organisation, Chandigarh. Currently, he is working as an Assistant Professor at the School of Computer Science and Engineering of Shri Mata Vaishno Devi University, Katra, Jammu and Kashmir. His area of interest is image processing and computer vision. He has authored many SCI journal publications and filed a few patents in India and USA. He is the reviewer of many SCI journals and also received the funding for the sponsored project from the government funding agencies. 


\section{Introduction}

Plants are used as food. Our Indian economy mainly depends upon agriculture. Many plant species had disappeared and many are at the verge of disappearing (Darshana et al., 2015). Many computerised systems had developed for both classifications as well as for identification of plants. To identify the plant it is important to know its characteristics and its leaf structure. Morphological structures are the same for the different leaves. There are different features present in each leaf. It is important to identify and differentiate the features of leaves by segmenting the leaf from its background. Leaf segmentation is the process of dividing a leaf image into small segments. Segmentation process should be done in a way so that important features present in the leaf can be extracted without changing the morphological appearance. Traditionally, the leaf segmentation task was carried out by the experts that consume a large amount of time. In the automatic leaf segmentation approach, the leaf is closely monitored through digital cameras or time of flight (ToF) cameras to extract the phenotypic characteristics of each leaf. The phenotype of the plant describes the features of the plant that can be seen through naked eyes such as the height of the leaf, margin, shape, colour of leaf, etc. By examining the phenotypic characteristics of a plant it would be easy to examine different symptoms of the plant diseases (Darshana et al., 2015). There are various techniques used for the segmentation of leaf, i.e., artificial neural network (ANN) (Minervini et al., 2016) histogram colour space (Barbedo, 2016), etc.

Leaf Segmentation has to deal with various difficulties as in the case of the illumination conditions where leaf images can contain the shadow of the dark and light areas that occurred due to different lighting conditions. Feature extraction and leaf segmentation algorithm should be developed in a way that automatically removes the unwanted shadows from the leaf image. In manual segmentation, leaf growth and leaf area were detected by an individual. Many biologists grow plants of Arabidopsis and tobacco plant and record their phenotypic traits manually. Many experts had agreed that due to lacking of automatic algorithm it was very difficult to extract the finely grained data from the different dataset as there is a requirement of a large amount of time and good concentration for recording and tracking of the leaf area and leaf growth of a plant.

Now a day, many algorithms had developed for the measurement of the plant features of any leaf size (Singh et al., 2016). In the case of the rosette plant (An et al., 2016), plant projected leaf area (PLA) is considered as a good approximation for the plant size. As plants are dynamic in nature and every day it grows and their properties change most of the time during their growth and it is difficult to keep an eye on it. Therefore a method or technique should be developed for the automatic monitoring and segmentation of the leaf.

Leaf segmentation is performed with a few objectives such as to count the number of leaves in a plant as some leaves are overlapped (Minervini et al., 2016), to detect chlorophyll pigment in each leaf (Alenya et al., 2012), to calculate the rosette area and leaf length (An et al., 2016), to detect different colour spots in the affected area of leaf (Barbedo, 2016), etc. 
Table 1 The methodologies adopted in literature for the monitoring of leaf segmentation

\begin{tabular}{|c|c|c|c|c|c|c|}
\hline 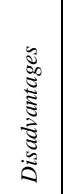 & 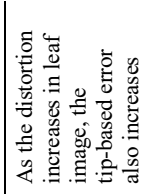 & 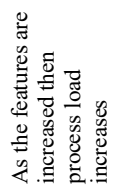 & 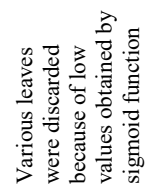 & 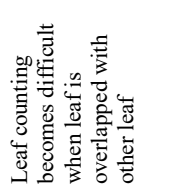 & 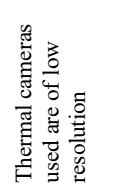 & 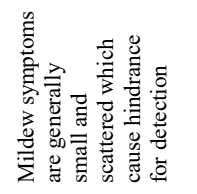 \\
\hline 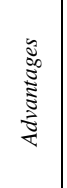 & 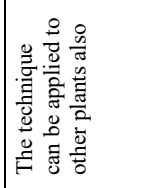 & 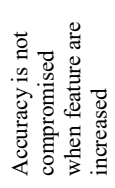 & 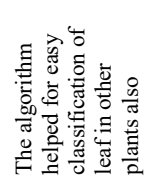 & 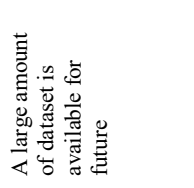 & 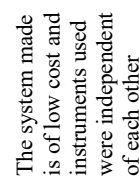 & 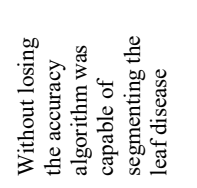 \\
\hline & 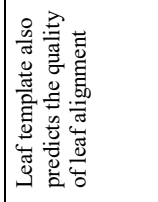 & 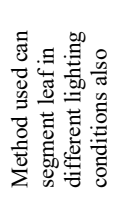 & 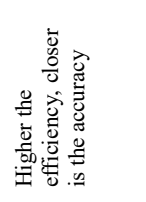 & 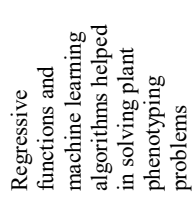 & 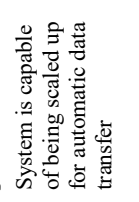 & 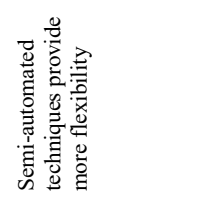 \\
\hline 节 & 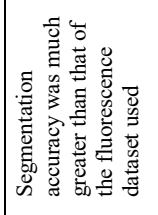 & 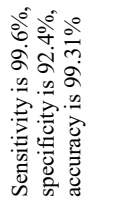 & 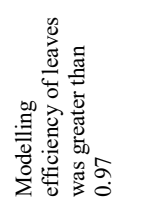 & 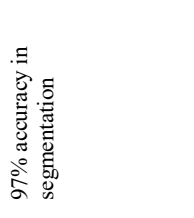 & 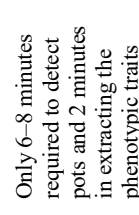 & 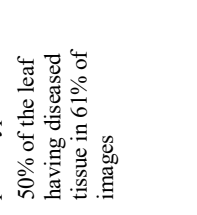 \\
\hline 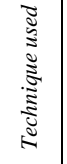 & 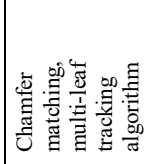 & 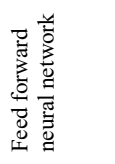 & 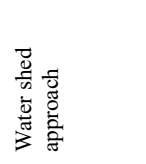 & 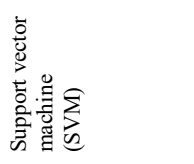 & 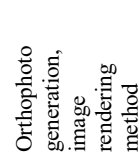 & 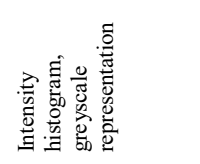 \\
\hline 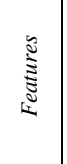 & 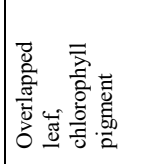 & 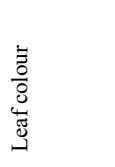 & 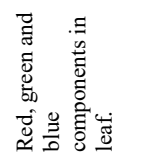 & 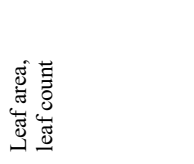 & 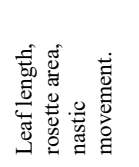 & 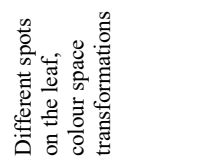 \\
\hline 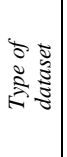 & 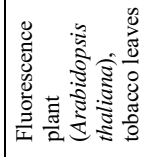 & & 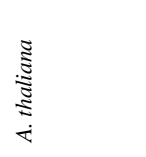 & 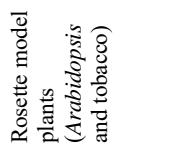 & 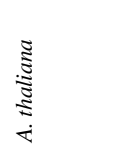 & 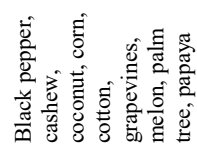 \\
\hline 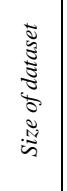 & 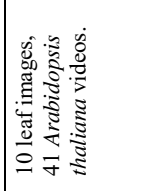 & 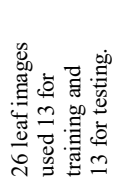 & 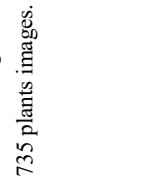 & 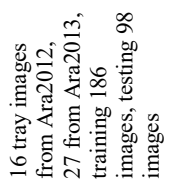 & 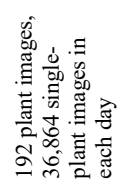 & $\begin{array}{l}\mathscr{8} \\
\stackrel{0}{0} \\
\stackrel{\Xi}{\Xi} \\
\infty \\
\sigma\end{array}$ \\
\hline 芯 & 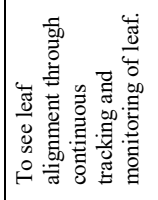 & 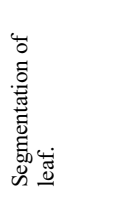 & 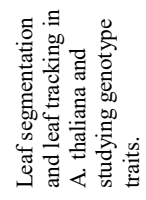 & 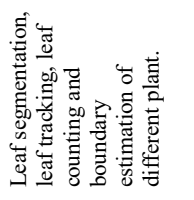 & 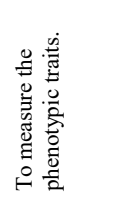 & 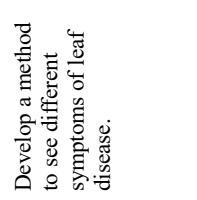 \\
\hline 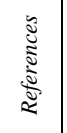 & 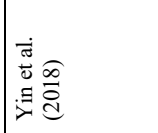 & $\begin{array}{l}\text { 恙突 } \\
\text { 。 }\end{array}$ & 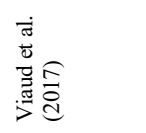 & 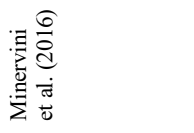 & 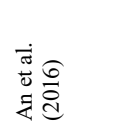 & 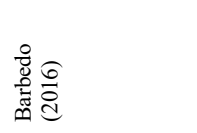 \\
\hline$\dot{v i} \stackrel{\dot{q}}{ }$ & $\mid-$ & $\sim$ & $m$ & + & is & \\
\hline
\end{tabular}


Table 1 The methodologies adopted in literature for the monitoring of leaf segmentation

\begin{tabular}{|c|c|c|c|c|c|}
\hline 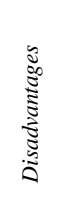 & 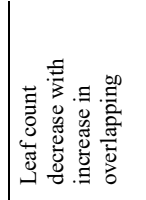 & 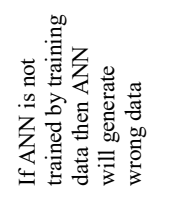 & 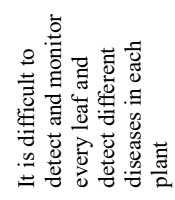 & 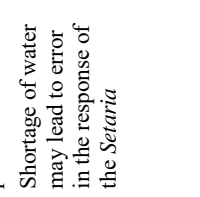 & 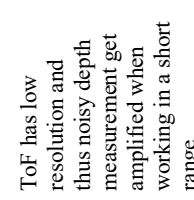 \\
\hline $\begin{array}{l}\mathscr{\tilde { J }} \\
\mathbb{\Xi} \\
\tilde{\Xi} \\
\dot{z}\end{array}$ & 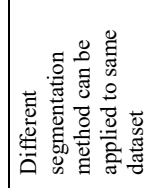 & 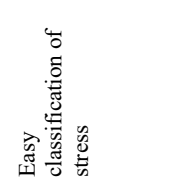 & 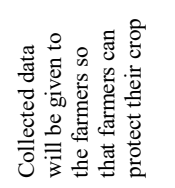 & 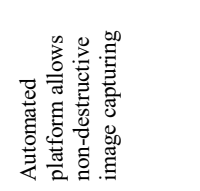 & 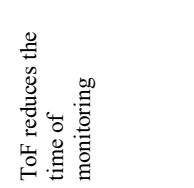 \\
\hline 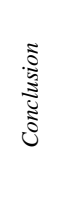 & 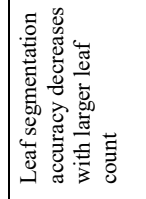 & 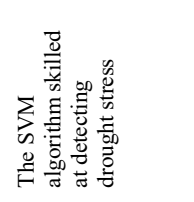 & 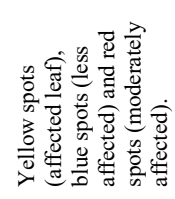 & 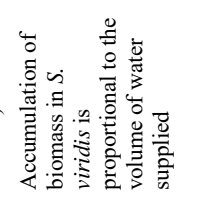 & 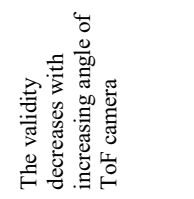 \\
\hline 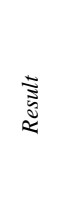 & 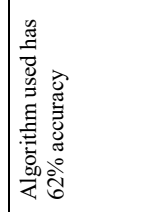 & 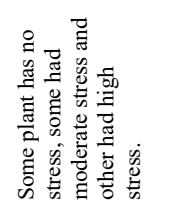 & 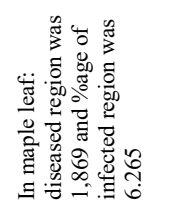 & 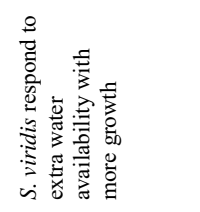 & 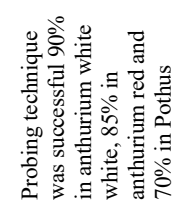 \\
\hline 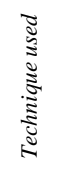 & 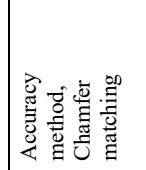 & 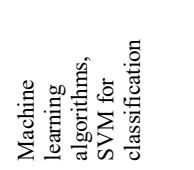 & 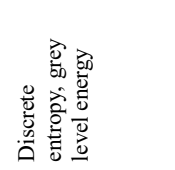 & 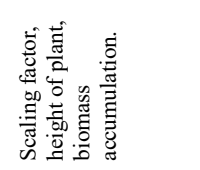 & 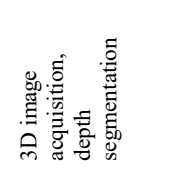 \\
\hline 芯 & 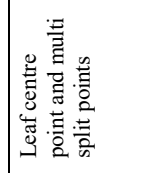 & 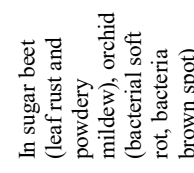 & 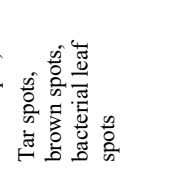 & 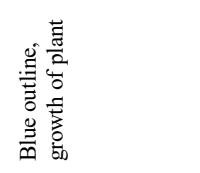 & 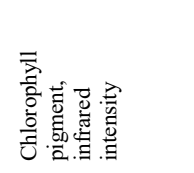 \\
\hline 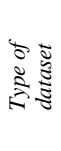 & 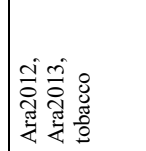 & 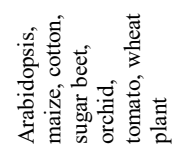 & 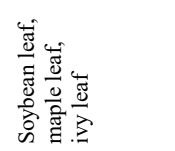 & 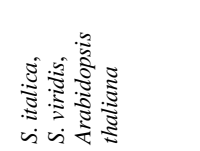 & 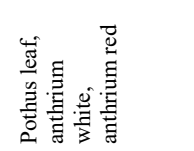 \\
\hline 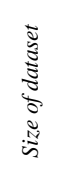 & 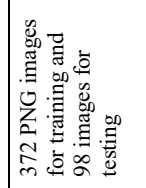 & 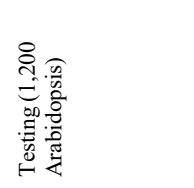 & 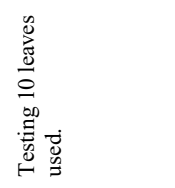 & 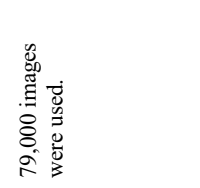 & 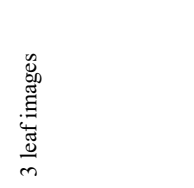 \\
\hline 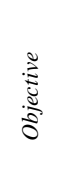 & 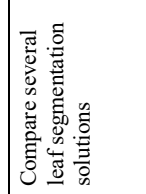 & 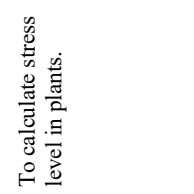 & 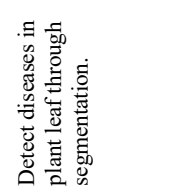 & 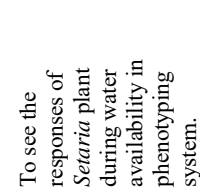 & 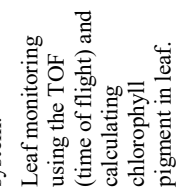 \\
\hline$\frac{\mathscr{\Xi}}{\check{\Xi}}$ & 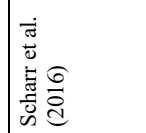 & 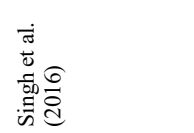 & 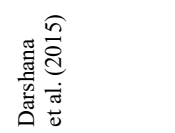 & 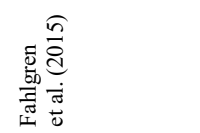 & 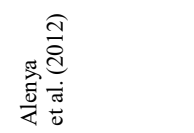 \\
\hline$\dot{\varphi} \dot{8}$ & 1 & $\infty$ & $a$ & 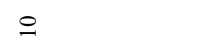 & \\
\hline
\end{tabular}


Various methods (Arnal Barbedo, 2013) like the Otsu method (Ozturk, 2017) which is simple to implement, k-means clustering (Valliammal and Geethalakshmi, 2012) which helps in the detection of the homogenous data in the leaf image, neural network which helps in feature extraction from the segmented leaf which includes training of machine. A test image is an input to the machine to evaluate whether it is able to identify the appropriate class based on the features of the test image or not. Table 1 demonstrates methodologies adopted in literature for monitoring of leaf.

For the automatic segmentation of leaf, feature extraction methods were adopted for leaf images (Ozturk, 2017) and sensitivity, specificity and accuracy were computed for the validation of different methods. The sensitivity, specificity, and accuracy were reported as $99.6 \%, 92.4 \%$, and $99.31 \%$ respectively. The advantage of using feature extraction methods is that accuracy does not change when features are increased. As features are increased the processing load also increases which is a demerit of feature extraction methods. Some rosette plant's leaves, pothus leaves were used for the segmentation process and regressive functions (Minervini et al., 2016) were evaluated. For this various machine learning algorithms (Singh et al., 2016) were used and segmentation accuracy was calculated, i.e., $67 \%$ of images contain no leaf counting error. Leaf counting is difficult as some leaves are overlapped and can cause hindrance in leaf count. Probing technique was $90 \%$ successful in case of the anthurium white, $85 \%$ successful in case of the anthurium red and $70 \%$ in case of pothus and time of flight camera used has succeeded in probing of about $82 \%$.

The ToF camera (Alenya et al., 2012) was used for monitoring of leaf. The results were obtained more accurate with ToF camera as it provided the detail information about the leaf growth and chlorophyll pigment in the leaf, etc. ToF camera has one disadvantage as it has low resolution and its noisy depth measurement is increased when the process is of short range. Some other features were also seen such as Tar spots, brown spots (Darshana et al., 2015), the area of the diseased leaf, the percentage of the infected area. Entropy (Zhao et al., 2015) was calculated in each leaf image with is calculated as the uncertainty in the information of the source image and information redundancy between the images. In some leaf images, firstly orthophoto is generated and then image rendering method (An et al., 2016) (photorealistic and non-photo realistic image from 2D or $3 \mathrm{D}$ image) was used for the pre-processing of leaf image. High performance computing (HPC) uses 6-8 minutes to detect spots and require 2 minutes to extract the traits in the leaf image. Thermal cameras were also used for monitoring and tracking of leaf, the advantage of using thermal cameras is that it does not need light to capture high contrast images and can monitor plants in darkness also. For the segmentation process some techniques were used such as chamber matching (Fahlgren et al., 2015), local search method (Yin et al., 2018) and leaf tracking algorithm for solving the optimisation problem present in each leaf that mainly depends on the template transformation. Water shed approach (Viaud et al., 2017) is also used in some leaf segmentation process and then features were extracted, various leaves were discarded because the values obtained by the sigmoid function were less than the threshold. In some cases leaf image is converted into greyscale representation and histogram peak (Barbedo, 2016) is developed and concluded that greater the height of the peak, healthier the leaf and if peak is small then symptoms of disease is present on leaf and $83 \%$ cases the technique used was capable of detecting the disease symptoms. In some cases, feature extraction phase is used to detect leaf centre points and multi split points (Scharr et al., 2016) for the 
corresponding leaf segmentation. From the leaf segmentation process, it was concluded that larger the leaf count lesser will be the segmentation accuracy.

In some leaf images SVM algorithm (Singh et al., 2016) used for detecting the stress level in leaf through machine learning technique (Singh et al., 2016). The method used was biomass accumulation, blue outliner on the boundary of leaf to see the response of Setaria plants. Plant CV (Fahlgren et al., 2015) was used to calculate the growth of the plant and it was found that $S$. virdis showed more growth than S. italian and shortage of water can lead to incorrect results. An automatic method was developed which automatically identify the leaf and this automatic method was k-means, Chan-Vese algorithm (Raji and Thyagharajan, 2015), etc. It was found that Chan-Vese algorithm provides good result for both single as well as the bunch of leaves. The only disadvantage of Chan-Vese algorithm is this algorithm provides less information about the shape of the leaf.

\section{Material and methods}

Material and methods include the techniques to be used for the segmentation of leaf. There are different steps as listed in Figure 1. Leaf image acquisition means the image is taken from which source, i.e., images taken from camera or through the video. Leaf image pre-processing involves removal of the noise from the leaf image which may be in the form of dust, distortion, etc. After the removal of noise from image, thereafter features are extracted from the leaf and segmentation process is applied, i.e., image is divided into small region which is done by applying various methods such as k-means (Valliammal and Geethalakshmi, 2012; Baghel and Jain, 2016), ANN (Singh et al., 2016), threshold segmentation (Zhao et al., 2015), etc.

Figure 1 Steps required for leaf segmentation

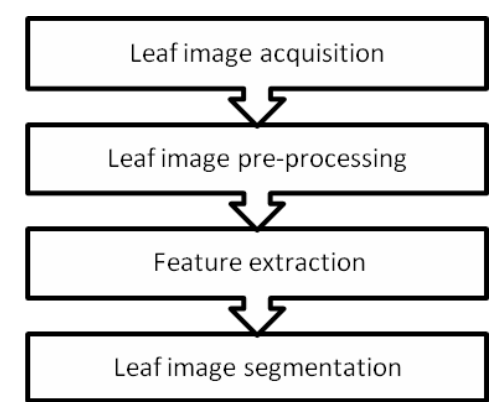

\subsection{Leaf image acquisition}

Digital acquisitions mean digital encoding for the visual representation of an image which includes the interior structure of the image. It is broadly defined as the action of retrieving an image from a source usually hardware-based source so that image can be passed through whatever process needs to be worked afterward. There are different factors that are involved in the image acquisition, i.e., setting up a system for long terms and maintenance of hardware for growing plants and a device that can be camera which will capture different images of leaf and if not properly configured the visual defects can 
be produced which complicate the process of segmentation. An improper setup may produce images of low quality which can't be segmented using a deeper process also.

The real-time acquisition usually involves the retrieving the image from the real source and creates a stream of files that can process automatically and are converted into a single format. Some the images are taken from the environment which contains noise such as the soil and the background regions such as the dark region and the shadows in the images. The dark region and shadows in the image may cause failure to segmentation process. In case of 3D images (Ozturk, 2017) series of plant leaf images were taken through different cameras aligned over to the leaf and ToF cameras are used for the record the measurements of the image. The camera emits infra light which calculates the distance between the emitted light and the light which is reflected back by the leaf. ToF camera has two drawbacks as the image captured is of low resolution and noisy depth measurements (Ozturk, 2017). There are two filters used within TOF camera, i.e., jump edge filter and an averaging filter to remove the noise and depth errors.

A sensing system was set up where plants are grown and are monitored through camera sensors (Minervini et al., 2016) which will capture the images of the leaf containing features such as the growth of leaf, leaf area, etc. and these images were directly sent to the other computer system via a wireless connection. Environmental conditions were provided to the growing plants in the sensing system so that plants can grow as they grow in a natural environment. Some images were taken from Canon DSLR cameras and this camera has two flashes with diffusers to create an illumination condition. Colour dot system (An et al., 2016) was also used to track the growing period of the plant. Many images of the Arabidopsis thaliana were taken using the phenoscope which is an automatic phenotyping platform and from there 21 images are considered which is of different genotype and all other plants were grown in the same environment and some images are taken on 9th day after sowing. For evaluation, four genotypes were considered and some images are captured using the analogue digital cameras also which contain red luminous light so that pixels are clear and are stored in the computer memory (Arnal Barbedo, 2013) for further processing.

\subsection{Leaf image pre-processing}

Pre-processing method is used for low quality or low level images that involve the improvement of images and removes the unwanted distortion present in the image in order to enhance the image so that important features of the leaf image could be extracted for further processing. Figure 2 explained about the pre-processing steps, i.e., firstly plant's image is converted into colour space then noise removal takes place, i.e., removal of dust or shadow which make the segmentation process easy, after the noise removal, image enhancement takes place like increasing the contrast, sharpness of leaf image so that surface spots are clear.

There are three categories for the image pre-processing technique:

a geometric transformations

b pixel brightness transformation

c image restoration. 
Figure 2 Steps for pre-processing of plant's image

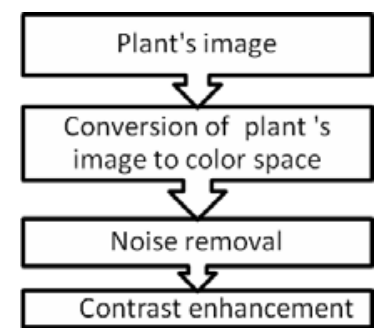

Geometric transformation involves the removal of the geometric distortion which had occurred during the capture of the image. Pixel brightness (Arnal Barbedo, 2016) of image means increasing the brightness of image so that leaf image is clear and it could be easy to see the features on the leaf surface and image restoration is the recovery of the image from a degraded version (noisy image or blurry image) to a high-resolution image for that it is important to have knowledge about the entire image. Before the pre-processing it is important to have knowledge about the image acquisition as if under which condition the image is captured, Knowledge about the general properties of the leaf image and knowledge about the features to be searched and the segmentation process techniques to be used.

The images pre-processing include the removal of distortion from the image, dust from the leaf surface, removal of dark regions and shadows from the image which had occurred during image capturing and if image pre-processing is not done correctly can make segmentation process more complicated. As in case of $3 \mathrm{D}$ image acquisition (Alenya et al., 2012) more than two cameras were used to take a raw image. Image pre-processing was done for colour correction (Valliammal and Geethalakshmi, 2012) and to reduce image optical distortion. In the colour correction technique (Singh and Misra, 2017), a white balance operation is used to pre-process the raw image. Due to the lens distortion, the accuracy of the measurement of the leaf is reduced and if the leaves are overlapped then it is not possible to extract features from that different leaves so overlapped leaf should be rotated in a particular angle so that some features of the other leaves could be extracted.

\subsection{Feature extraction}

Feature extraction starts from measuring the data and deriving the values from the leaf image. When a dataset is very large then input data is transformed into its feature set. The feature set will contain all the relevant information about the image. Basically, feature extraction involves reducing the amount of pixel to describe a large amount of data and involves the combination of the pixel to describe data with sufficient accuracy. There are various techniques for the feature extraction such as dimensionality reduction, i.e., principal component analysis (Singh et al., 2016) and independent component analysis (Singh et al., 2016). Feature extraction involves the extraction of important features from the dataset. 


\subsubsection{Feature-based extraction}

Chlorophylls pigment and infrared intensity reflected by leaf were calculated after the pre-processing step from Anthurium red and Pothos plant leaf image and in maple leaf image features like leaf area contain tar spots and in case of sugarcane and rice leaf red spots and rust on the surface of leaf were detected during the feature extraction process. Thereafter in maple leaf, Anthurium red leaf, pothus plant leaf and sugarcane leaf feature-based segmentation process was applied.

\subsubsection{Knowledge-based extraction}

In Arabidopsis thaliana plant leaf images, leaf alignment, increase in leaf area were observed and by applied segmentation process it was concluded that when a threshold is increased, large tip-based errors are considered which contribute to the large landmark error and tracking inconsistency. In tobacco leaf image, from the shape of leaf boundaries and leaf counting and it was concluded that the segmentation process decreases with an increase in the leaf count because of high overlapping between the mature and young leaf.

\subsubsection{Performance-based extraction}

Setaria viridis (green millet), S. italica are the drought-tolerant crops where wild Setaria maintains efficient growth using water while domesticated Setaria uses less water usage for efficient growth. During water availability, $S$. viridis plants grow taller than the $S$. italica and $S$. viridis showed more growth of about $0.6 \mathrm{~g}$ /day than that of $S$. italica (Fahlgren et al., 2015).

\subsection{Leaf segmentation}

Leaf segmentation is the process of partitioning a leaf image into multiple segments that share similar attributes (like colour, texture) to making it more useful for the analysis and interpretations. Leaf segmentation is done in order after features extraction process in leaves like to calculate genotype trait, chlorophyll content, leaf area including infected area, etc. The segmentation process is the main step in the image analysis. Image segmentation is based upon two properties, i.e., similarity and dissimilarity. Similarity means dividing the image into different regions based on similar properties (Patil and Kumar, 2011). Discontinuity means dividing the image into different regions based on the intensity level of the image (Patil and Kumar, 2011). Discontinuity approach can be classified into three types such as the line detection, point detection, and edge detection approach. In edge detection is mainly used for segmenting the leaf images based on the local changes in the intensity. Edge detection is of three types (Kumar, 2012), i.e., step, roof and ramp edge. Step edge involves the changes between the two intensity levels over the distance of one pixel as in case of computer-generated images. Roof edge determines the thickness and sharpness of the leaf and ramp edges (Chaki et al., 2015) mainly seen when the change in the intensity of leaf images is not instantaneous. Line detection consists of convolution kernels (Chaki et al., 2015) which is used detect particular lines of a particular width and point detect is used to detect point features in an image and these features are extracted from the region where there is significant edge strength. 
There are two main methods used for image segmentation such as the region based segmentation (Kamlapurkar, 2016) and clustering-based segmentation (Kamlapurkar, 2016). Region-based segmentation leaf region is segmented based on the intensity information and this region is added to the existing region of the leaf and intensity information is taken from the input image which will be used for the region growing segmentation. In region merging segmentation (Kamlapurkar, 2016) the leaf image is divided into different blocks and these blocks are again sub-blocks and from the sub-blocks a seed point (centroid) is calculated (Singh et al., 2016). And this process repeated until no region is present in the neighbour. In region splitting, maximum and minimum intensity values of the leaf image are calculated and if these values are less than the threshold value of the leaf then leaf region is spat. Clustering-based segmentation (Valliammal and Geethalakshmi, 2012) includes mean shift segmentation and k-means segmentation (Baghel and Jain, 2016). In the means shift segmentation, every feature set in the leaf image is supposed to be a cluster set and radius is calculated for each dataset and from that radius a centre is made on the basis of the mean of data point and the process will continue till all the data points converges at a single centre and in case of k-means clustering (Valliammal and Geethalakshmi, 2012) the leaf image is divided into different clusters and one of the cluster will contain disease features (Larese et al., 2014).

\section{Result}

Various methods were reviewed for automatic leaf segmentation of plant. It was found that different methods show different efficiency and accuracy results based upon the features extraction and segmentation techniques. Methods were compared with other methods to compute other important features and success rate of different segmentation techniques.

\section{Discussion}

Itakura and Hosoi (2018) captured leaf images from Canon EOS M2 in a horizontal position. The objective was to extract a feature from the leaf and apply the segmentation method. 3D leaf images were pre-processed using Agisoft photo scan software and leaf area is the feature extracted. Water shed algorithm (Fahlgren et al., 2015) with attributes expanding method and with the simple projected method is applied for leaf segmentation. It was found that leaf segmentation success rate was $86.9 \%$. Table 2 represents the few examples demonstrating the methods used against the generalised steps involved for leaf segmentation process. Singh and Misra (2017) acquire leaf images from a digital camera. Their objective was to detect different colour spots on the leaf surface and apply the segmentation method. Thereafter, smoothing filter (capture important patterns in data and leaving out the noise) and clipping method (the only important region is cropped) used for the pre-processing of the leaf image. Tactile texture and different colour spots on leaf surface are the features extracted from the leaf. A genetic algorithm was used to segment the cluster components of leaf image during the leaf segmentation. 
Table 2 A few examples demonstrating the methods used against the generalised steps involved for the leaf segmentation process

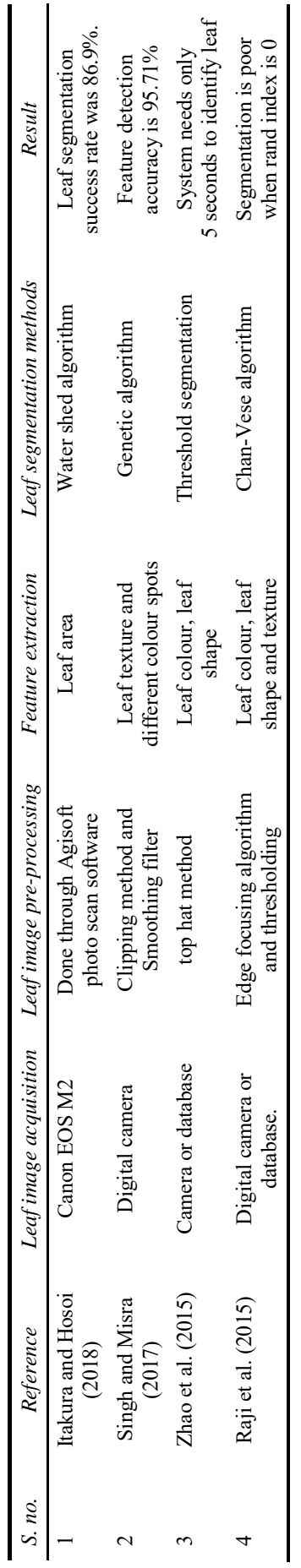


Zhao et al. (2015) captured the plant's leaf images from the camera or from already exist local database. The objective was to make a system for automatic plant identification. Top hat method was used for pre-processing of leaf images. Leaf colour and leaf texture were the features extracted from the leaf images, thereafter thresholding segmentation method was applied in which RGB (red, green, blue) colour space was converted into greyscale and for feature extraction different method were used such as PHOG (pyramid histogram of gradient) in which shape and appearance of local object within the image is described by intensity gradient distribution, colour HSV (hue, saturation, value) and wavelet. The system was trained with training and testing data to identify the plant's leaf images and after training the system it was found that the system takes only 5 seconds to identify the plant. After training the identification accuracy was increased from 0.76 to 0.89. Raji and Thyagharajan (2015) captured the leaf images from digital cameras or already existing local database. The main objective was to identify the best method for segmenting the leaves images from complex background Edge focusing algorithm (remove less relevant information from leaf image) and thresholding method were used for pre-processing of leaf images. Leaf colour, texture and leaf shape were the features extracted after the pre-processing step. Chan-Vese algorithm and K-means algorithm was used for the segmentation of herbal leaves based on the minimum energy states from the corresponding pixels. Chan-Vese algorithm worked well for thin leaf edge and thus required less time for execution. Rand index was calculated based on the segmentation process and it was found that leaf segmented through Chan-Vese algorithm had high value of rand index than leaf segmented through K-means algorithm (Valliammal and Geethalakshmi, 2012; Baghel and Jain, 2016). Thus segmentation efficiency was more in case of Chan-Vese algorithm than k-means clustering (Baghel and Jain, 2016).

\section{Conclusions}

This paper presents a review of the methods of automatic segmentation of plant leaves. The acquired images were pre-processed by using different methods such as clipping method, smoothing method, top hat method, edge focusing method, etc. and a number of features are extracted from them using different methods like SVM, machine learning algorithms, etc. The machine learning algorithms were used in various training methods to predict various stress level and to calculate leaf area in a different plant. SVM algorithms are also skilled at detecting drought stress also. With the leaf boundary and leaf structure information, photosynthetic efficiency can also be computed. Effective methods are used for segmentation of leaf under different lighting conditions as in this case, the artificial neural network is trained. It can be concluded by reviewing various methods that as the efficiency of methods increases then the accuracy of getting accurate results also increases and leaf segmentation accuracy decreases with larger leaf count.

\section{Acknowledgements}

The authors would like to acknowledge the work completed under the project grant received from the University Grant Commission through UGC-start-up Grant under RP-103. 


\section{References}

Alenya, G., Dellen, B., Foix, S. and Torras, C. (2012) 'Robotic leaf probing via segmentation of range data into surface patches', IROS Workshop on Agricultural Robotics: Enabling Safe, Efficient, Affordable Robots for Food Production, Vilamoura, Portugal, pp.1-6.

An, N., Palmer, C.M., Baker, R.L., Markelz, R.J.C., Ta, J., Covington, M.F., ..., Weinig, C. (2016) 'Plant high-throughput phenotyping using photogrammetry and imaging techniques to measure leaf length and rosette area', Computers and Electronics in Agriculture, Vol. 127, pp.376-394 [online] https://doi.org/10.1016/j.compag.2016.04.002.

Arnal Barbedo, J.G. (2013) 'Digital image processing techniques for detecting, quantifying and classifying plant diseases', SpringerPlus, Vol. 2, No. 1, [.660, doi: 10.1186/2193-1801-2-660.

Arnal Barbedo, J.G. (2016) 'A review on the main challenges in automatic plant disease identification based on visible range images', Biosystems Engineering, Vol. 144, pp.52-60, ScienceDirect [online] https://doi.org/10.1016/j.biosystemseng.2016.01.017.

Baghel, J. and Jain, P. (2016) 'K-means segmentation method for automatic leaf disease detection', International Journal of Engineering Research and Application, Vol. 6, No. 3, pp.83-86.

Barbedo, J.G.A. (2016) 'A novel algorithm for semi-automatic segmentation of plant leaf disease symptoms using digital image processing', Tropical Plant Pathology, Vol. 41, No. 4, pp.210-224 [online] https://doi.org/10.1007/s40858-016-0090-8.

Chaki, J., Parekh, R. and Bhattacharya, S. (2015) 'Plant leaf recognition using texture and shape features with neural classifiers', Pattern Recognition Letters, Vol. 58, pp.61-68 [online] https://doi.org/10.1016/j.patrec.2015.02.010.

Darshana, A., Majumdar, J. and Ankalaki, S. (2015) 'Segmentation method for automatic leaf disease detection', International Journal of Innovative Research in Computer and Communication Engineering, Vol. 3, No. 7, pp.7271-7282.

Fahlgren, N., Feldman, M., Gehan, M.A., Wilson, M.S., Shyu, C., Bryant, D.W., ..., Baxter, I. (2015) 'A versatile phenotyping system and analytics platform reveals diverse temporal responses to water availability in setaria', Molecular Plant, Vol. 8, No. 10, pp.1520-1535 [online] https://doi.org/10.1016/j.molp.2015.06.005

Itakura, K. and Hosoi, F. (2018) 'Automatic leaf segmentation for estimating leaf area and leaf inclination angle in 3D plant images', Sensors, Vol. 18, No. 10, p.3576 [online] https://doi.org/10.3390/s18103576.

Kamlapurkar, S.R. (2016) 'Detection of plant leaf disease using image processing approach', International Journal of Scientific and Research Publications, Vol. 6, No. 2, pp.73-76.

Kumar, R. (2012) 'Image segmentation using discontinuity-based approach', June, Vol. 2, Nos. 1/2, pp.72-78.

Larese, M.G., Namías, R., Craviotto, R.M., Arango, M.R., Gallo, C. and Granitto, P.M. (2014) 'Automatic classification of legumes using leaf vein image features', Pattern Recognition, Vol. 47, No. 1, pp.158-168 [online] https://doi.org/10.1016/j.patcog.2013.06.012.

Minervini, M., Fischbach, A., Scharr, H. and Tsaftaris, S.A. (2016) 'Finely-grained annotated datasets for image-based plant phenotyping', Pattern Recognition Letters, Vol. 81, pp.80-89 [online] https://doi.org/10.1016/j.patrec.2015.10.013.

Ozturk, B.A.S. (2017) 'Automatic leaf segmentation using grey wolf optimizer based neural network', Paper presented at the 2017 Electronics Conference, DOI: 10.1109/ ELECTRONICS.2017.7995228

Patil,, J.K. and Kumar, R. (2011) Advances in Image Processing for Detection of Plant Diseases, Vol. 2, No. 2, pp.135-141.

Raji, I.K. and Thyagharajan, K.K. (2015) 'An analysis of segmentation techniques to identify herbal leaves from complex background', Procedia - Procedia Computer Science (ICCC 2014), Vol. 48, pp.589-599 [online] https://doi.org/10.1016/j.procs.2015.04.140. 
Scharr, H., Minervini, M., French, A.P., Klukas, C., Kramer, D.M., Liu, X., ... Tsaftaris, S.A. (2016) 'Leaf segmentation in plant phenotyping: a collation study', Machine Vision and Applications, Vol. 27, No. 4, pp.585-606 [online] https://doi.org/10.1007/s00138-015-0737-3.

Singh, A., Ganapathysubramanian, B., Singh, A.K. and Sarkar, S. (2016) 'Machine learning for high-throughput stress phenotyping in plants', Trends in Plant Science, Vol. 21, No. 2, pp.110-124 [online] https://doi.org/10.1016/j.tplants.2015.10.015.

Singh, V. and Misra, A.K. (2017) 'Detection of plant leaf diseases using image segmentation and soft computing techniques', Information Processing in Agriculture, Vol. 4, No. 1, pp.41-49 [online] https://doi.org/10.1016/j.inpa.2016.10.005.

Valliammal, N. and Geethalakshmi, S.N. (2012) 'Plant leaf segmentation using non-linear K means clustering', International Journal of Computer Science Issues, Vol. 9, No. 3, pp.212-218.

Viaud, G., Loudet, O. and Cournède, P. (2017) 'Leaf segmentation and tracking in arabidopsis thaliana combined to an organ-scale plant model for genotypic differentiation', Frontiers in Plant Science, January, Vol. 7, pp.1-14 [online] https://doi.org/10.3389/fpls.2016.02057.

Yin, X., Member, X.L., Chen, J. and Kramer, D.M. (2018) 'Joint multi-leaf segmentation, alignment, and tracking for fluorescence plant videos', IEEE Transactions on Pattern Analysis and Machine Intelligence, Vol. 8828, No. CM, pp.1-14 [online] https://oi.org/10.1109/ TPAMI.2017.2728065.

Zhao, Z., Ma, L., Cheung, Y., Wu, X., Tang, Y., Lung, C. and Chen, P. (2015) 'Neurocomputing ApLeaf: an efficient android-based plant leaf identification system', Neurocomputing, Vol. 151, pp.1112-1119 [online] https://doi.org/10.1016/j.neucom.2014.02.077. 